\title{
Size distribution measurement and separation of ethanol cluster ion beams
}

\section{$\operatorname{AUTHOR}(\mathrm{S})$ :}

Ryuto, Hiromichi; Sugiyama, Kazumichi; Ozaki, Ryosuke; Kubo, Yudai; Takaoka, Gikan H.

\section{CITATION:}

Ryuto, Hiromichi ... [et al]. Size distribution measurement and

separation of ethanol cluster ion beams. Vacuum 2009, 84(5): 505-508

\section{ISSUE DATE:}

2009-12-10

URL:

http://hdl.handle.net/2433/89519

\section{RIGHT:}

This is not the published version. Please cite only the published version.; この論文は出版社版でありません。引用の際には出版社版を ご確認ご利用ください。 


\title{
Size Distribution Measurement and
}

\section{Separation of Ethanol Cluster Ion Beams}

\author{
Hiromichi Ryuto ${ }^{* 1}$, Kazumichi Sugiyama, Ryosuke Ozaki, \\ Yudai Kubo, and Gikan H. Takaoka
}

Photonics and Electronics Science and Engineering Center, Kyoto University Nishikyo, Kyoto 615-8510, Japan

\begin{abstract}
The distributions of the number of molecules per cluster (cluster size) of ethanol cluster ion beams were measured by the time-of-flight method. The cluster sizes at the peak and minimum tail (minimum size) of the distribution increased with increase of the retarding voltage. A difference appeared between the measured minimum size and the calculated values using a formula based on the early measurements on the velocity distributions of gas clusters.
\end{abstract}

Key words: cluster ion beam, size distribution TOF

PACS: 36.40.-c, 07.75.+h

\footnotetext{
* Corresponding author

Email address: ryuto@kuee.kyoto-u.ac.jp (Hiromichi Ryuto).

1 Formerly, H. Akiyoshi
} 


\section{Introduction}

The condensation phenomenon in an expanding nozzle flow was first mentioned by Becker et al. in 1956 [1], and organized into a cluster beam technique by Hagena et al. [2]. Clusters are aggregates of molecules cohered by van der Waals force with sizes ranging from dimers to thousands of monomers. The characteristic features of the interaction between cluster ion beams and solid surfaces, such as a high sputtering yield and surface smoothing effect [3], are mainly attributable to the high-density energy deposition and extremely low kinetic energy per molecule of the cluster ions that bombard the surface of the materials. The kinetic energy per molecule is inversely proportional to the number of molecules that compose a cluster (cluster size), and the number of molecules that bombard a certain small area is approximately proportional to the cluster size. Therefore, to control the cluster size is the key to control the irradiation effects of cluster ions on the surface of solid materials. Moreover, the radiation damage in silicon crystals caused by the cluster ion bombardment may be strongly related to the portion of the cluster ions that have high energies, which is in the small size part of the cluster size distribution. Therefore, to control the cluster size is also the key to reduce the radiation damage in silicon crystals. The reduction of the lattice disorders in silicon crystals is highly required in the production of high-density integrated circuits. In this article, cluster size distributions measured by the time-of-flight method are presented, and their dependences on the acceleration voltage and current of ionization electrons, and on the retarding voltage are discussed. 


\section{Experimental Procedure}

Figure 1 shows a schematic view of the liquid cluster ion beam apparatus. The apparatus consists of four vacuum chambers. A liquid container filled with ethanol is placed in the chamber positioned in the upstream part of the apparatus. The liquid container is heated to the temperature $T_{0}$ that corresponds to the required vapor pressure $p_{0}$ by a cable heater attached to the outer wall of the container. The temperature of the liquid container was maintained at $109^{\circ} \mathrm{C}$ that corresponded to the ethanol vapor pressure of 3 atm. The variation of the source temperature was approximately within $\pm 1^{\circ} \mathrm{C}$, which corresponded to the vapor pressure variation of $\pm 10 \mathrm{kPa}$. The vaporized ethanol is emitted to the chamber through a Laval nozzle. The temperature of the ethanol vapor decreases by adiabatic expansion, and ethanol clusters are formed [2]. The core part of the neutral cluster beam is selected using a skimmer whose skimmer opening is $1.3 \mathrm{~mm}$. The velocity distribution of the clusters produced by the adiabatic expansion method is reported to be highly uniform within $10 \%[2,4]$. It was reported that, to a good approximation, the cluster velocity is equal to the limiting velocity in an isentropic expansion [5]. By applying isentropic condition to Bernoulli's integral, and assuming that the pressure in the vacuum chamber is much smaller than $p_{0}$, we get [6],

$$
v_{0}=\sqrt{\frac{\gamma}{\gamma-1} \frac{2 k T_{0}}{m}}
$$

where $\gamma$ is the heat capacity ratio (1.13 for ethanol) and $m$ is the mass of the molecule. Therefore, the initial kinetic energies of the clusters are assumed to be approximately proportional to the cluster size $N$ to be $E_{0}=\frac{1}{2} N m v_{0}^{2}$. The remaining ethanol vapor is evacuated using a roots pump. The vacuum 
pressure of the vacuum chamber is the highest in the chamber that contains the liquid container positioned upstream part of the apparatus, and the lowest in the chamber for the target positioned downstream. The neutral clusters are ionized by the electrons emitted from a loop of a tungsten filament. The typical acceleration voltage $\left(V_{e}\right)$ and current $\left(I_{e}\right)$ of the ionization electrons are $200 \mathrm{~V}$ and $200 \mathrm{~mA}$, respectively. The ionized clusters are extracted by the extraction voltage typically from 3 to $9 \mathrm{kV}$. The monomers and the small-sized clusters that have low incident kinetic energies are eliminated by the retarding voltage $\left(V_{r}\right)$ applied to a retarding electrode. For example, the initial kinetic energy per molecule of the ethanol clusters produced with the vapor pressure of $3 \mathrm{~atm}$ is calculated using Eq. 1 to be $0.29 \mathrm{eV}$. Therefore, if a retarding voltage of 29 V was applied to the retarding electrode, only the cluster ions whose cluster sizes were larger than 100 would pass through the retarding electric field. After the elimination of the small clusters, the cluster ions are accelerated by an acceleration voltage $\left(V_{a}\right)$ applied to the acceleration electrode. The transverse divergence of the ethanol cluster ion beam is suppressed by an Einzel lens, and the beam is transported to a Faraday cup. A voltage of approximately $-300 \mathrm{~V}$ is applied to the electron suppressor of the Faraday cup.

The cluster size distribution was measured by the time-of-flight (TOF) method. Figure 2 shows a conceptual diagram of the cluster size measurement by the TOF method. A pulse of an ethanol cluster ion beam is produced by an electrostatic deflector, on which a high voltage pulse is applied. In this work, the beam current was measured using a Faraday cup instead of a micro-channel plate to measure the size distribution of intense cluster ion beams that had a sufficient intensity for the actual application in the semiconductor industry. The signal extracted from the Faraday cup is observed using a digital oscil- 
loscope. The time width of the pulse was typically $4 \mu \mathrm{s}$. The clusters have a kinetic energy $E=e V_{a}$, where e is the electric charge of the cluster ions. In the following discussion, we assume the singly charged ions. The velocity of the cluster ion is $v=\sqrt{2 E / M}$, where $M$ is the mass of the cluster ion. The calculation was made non-relativistically because the $\beta$ was approximately in the order of $10^{-5}$. The $M$ is approximately the product of the cluster size $N$ and the mass of an ethanol molecule $m$, because the binding energy of a cluster is small compared with the kinetic energy. The flight time between the deflector and the Faraday cup is

$$
t=L / v=L \sqrt{\frac{N m}{2 E}},
$$

where $L$ is the flight path length. Therefore, we can measure the distribution of the cluster size by the TOF method. The mass resolution of a linear TOF system is too low [7] to make a cluster-to-cluster measurement, but sufficient to know the shape of the distribution in general.

\section{Results and Discussion}

Figure 3 shows cluster size distributions of ethanol clusters measured with the acceleration voltages of ionization electrons from 100 to $500 \mathrm{~V}$. The vertical sizes were scaled for easy comparison. The variation in the peak position of the cluster size distribution with the increase of the $V_{e}$ was limited. It was reported that the ratio of multiply charged cluster ions increases with the increase in the $V_{e}$, and have a broad flat maximum approximately from 100 to $500 \mathrm{~V}$ [8]. The voltage range of the present measurement coincided with the flat part of the broad maximum. 
Figure 4 shows size distributions of ethanol clusters measured with the currents of ionization electrons from 50 to $500 \mathrm{~mA}$. The vertical sizes were scaled for easy comparison. The cluster size at the peak of the cluster size distribution slightly decreased with the increase in the $I_{e}$. It is possibly explained by the increase of the multiply charged ions with the increase in the $I_{e}$.

Figure 5 shows cluster size distributions measured with the retarding voltages from 27 to $109 \mathrm{~V}$. The cluster size at the peak of the cluster size distribution increased with the increase in the retarding voltage. Similarly, the cluster size at the minimum tail of the cluster size distribution (minimum size) increased with the increase in the retarding voltage. These results may indicate the efficiency of the retarding voltage method to eliminate the monomers and the small clusters.

Figure 6 shows a retarding voltage dependence of the minimum size. The line indicate the calculated value using Eq. 1. The minimum size measured at the retarding voltage of $0 \mathrm{~V}$ was normalized to be 0 . The calculated value deviated from the data points. The gradient of the calculated value against the retarding voltage was approximately 0.6 times of the gradient of the measured values. The effect of the dissociation caused by the Coulomb repulsion of multiply charged clusters may be limited to the cluster size below the critical size $[9,10]$, which is smaller than the cluster size discussed here. Therefore, it may be possible that the Eq. 1 is not applicable to the clusters of large molecules such as the ethanol clusters. The complexity of the ethanol molecule may be one of the possible cause of the difference between the data and the calculation using Eq. 1 assuming ideal gas. 


\section{Conclusion}

The cluster size distributions of ethanol cluster ion beams produced by the adiabatic expansion method were measured by the TOF method. The cluster size at the peak of the cluster size distribution slightly decreased with the increase in the current of ionization electrons. The cluster sizes at the peak and minimum tail of the cluster size distribution increased with the increase in the retarding voltage. However, a difference between the data and the calculation using Eq. 1 appeared in the gradient of the minimum sizes. The difference may suggest the limitation of the Eq. 1, which is based on the early measurements on the velocity distributions of gas clusters.

\section{References}

[1] E. W. Becker, K. Bier, W. Henkes, Z. Phys. 146 (1956) 333.

[2] O. F. Hagena, W. Obert, J. Chem. Phys. 56 (1972) 1793.

[3] I. Yamada, Nucl. Instrum. Methods. B 148 (1999) 1.

[4] O. F. Hagena, K. Varma, Rev. Sci. Instr. 39 (1968) 47.

[5] O. F. Hagena, in: P. P. Wegener (Ed.), Molecular Beams and Low Density Gas Dynamics, Dekker, New York, 1974, pp. 93-181.

[6] H. W. Liepmann and A. Roshko, Elements of Gasdynamics, John Wiley \& Sons, New York, 1960.

[7] R. B. Opsal, K. G. Owens, J. P. Reilly, Anal. Chem. 57 (1985) 1884.

[8] W. Obert, Int. J. Mass Spectrom. Ion Phys. 9 (1972) 295. 
[9] K. Sattler, J. Mühlbach, O. Echt, P. Pfau, E. Recknagel, Phys. Rev. Lett. 47 (1981) 160.

[10] J. G. Gay, B. J. Berne, Phys. Rev. Lett. 49 (1982) 194. 
Figure captions:

Fig. 1: Schematic view of liquid cluster ion beam apparatus.

Fig. 2: Conceptual diagram of time-of-flight measurement.

Fig. 3: Cluster size distributions measured with acceleration voltages of ionization electrons from 100 to $500 \mathrm{~V}$.

Fig. 4: Cluster size distributions measured with current of ionization electrons from 50 to $500 \mathrm{~mA}$.

Fig. 5: Cluster size distributions measured with retarding voltages from 27 to $109 \mathrm{~V}$.

Fig. 6: Retarding voltage dependence of cluster size at minimum tail of cluster size distribution. The circles indicate the data, and the line indicate the calculated value using Eq. 1. 


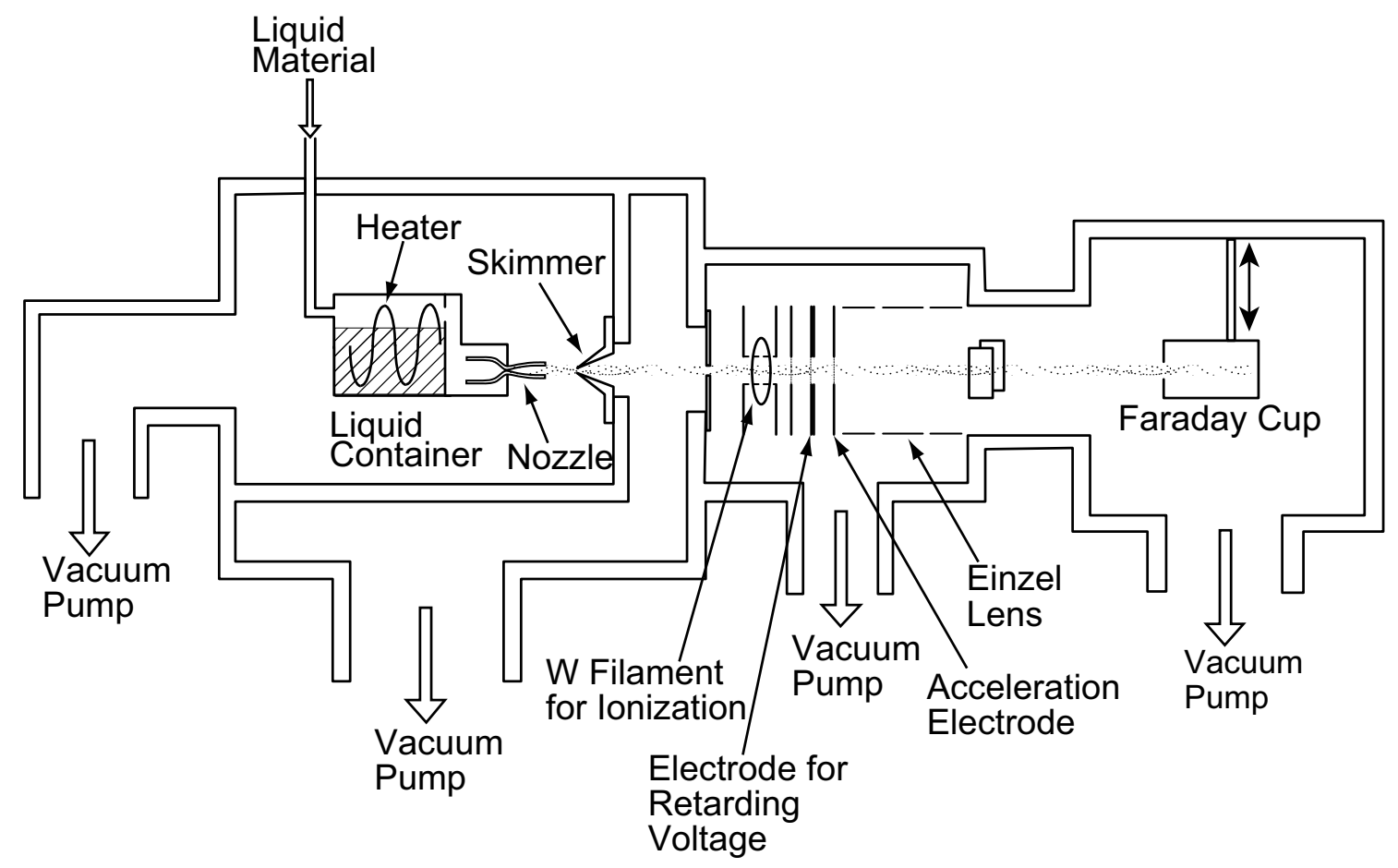

Fig. 1. 


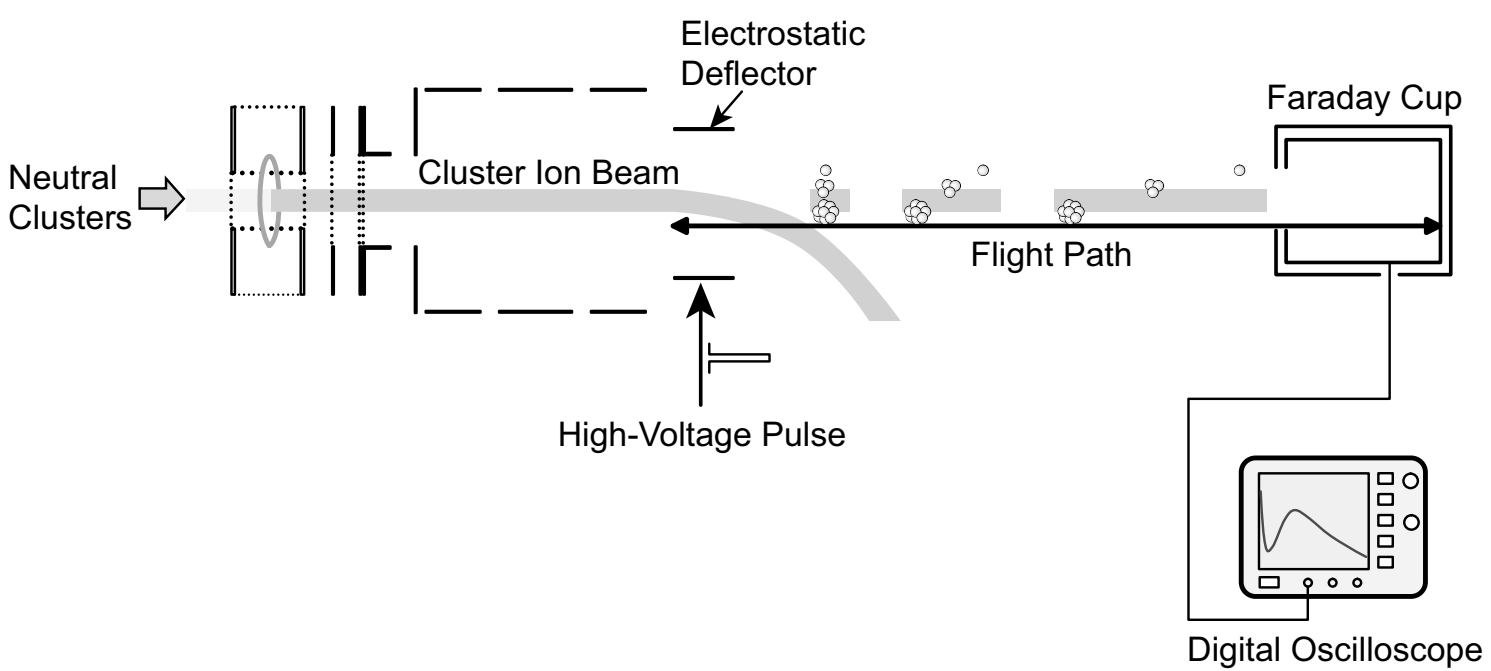

Fig. 2. 


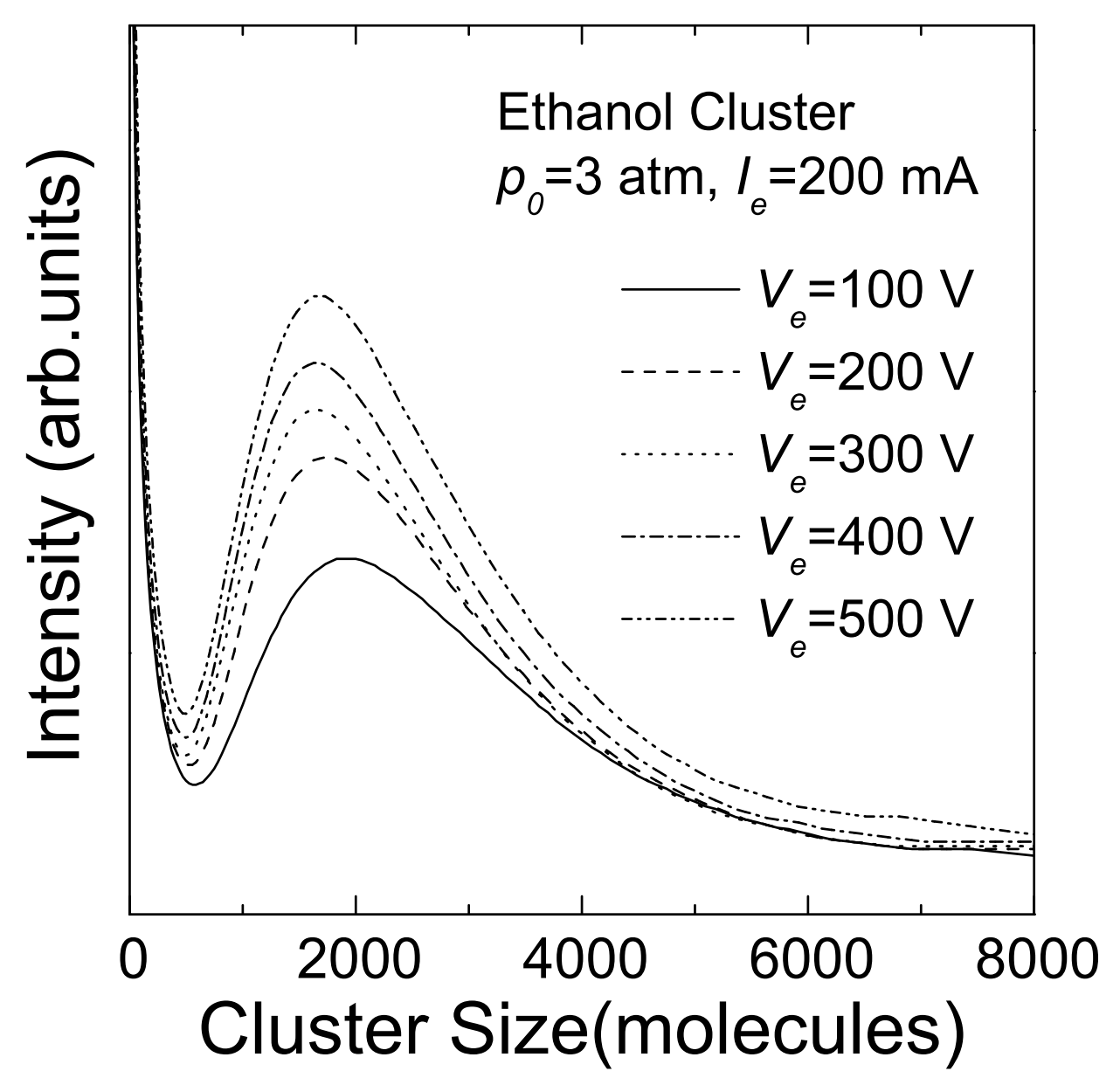

Fig. 3. 


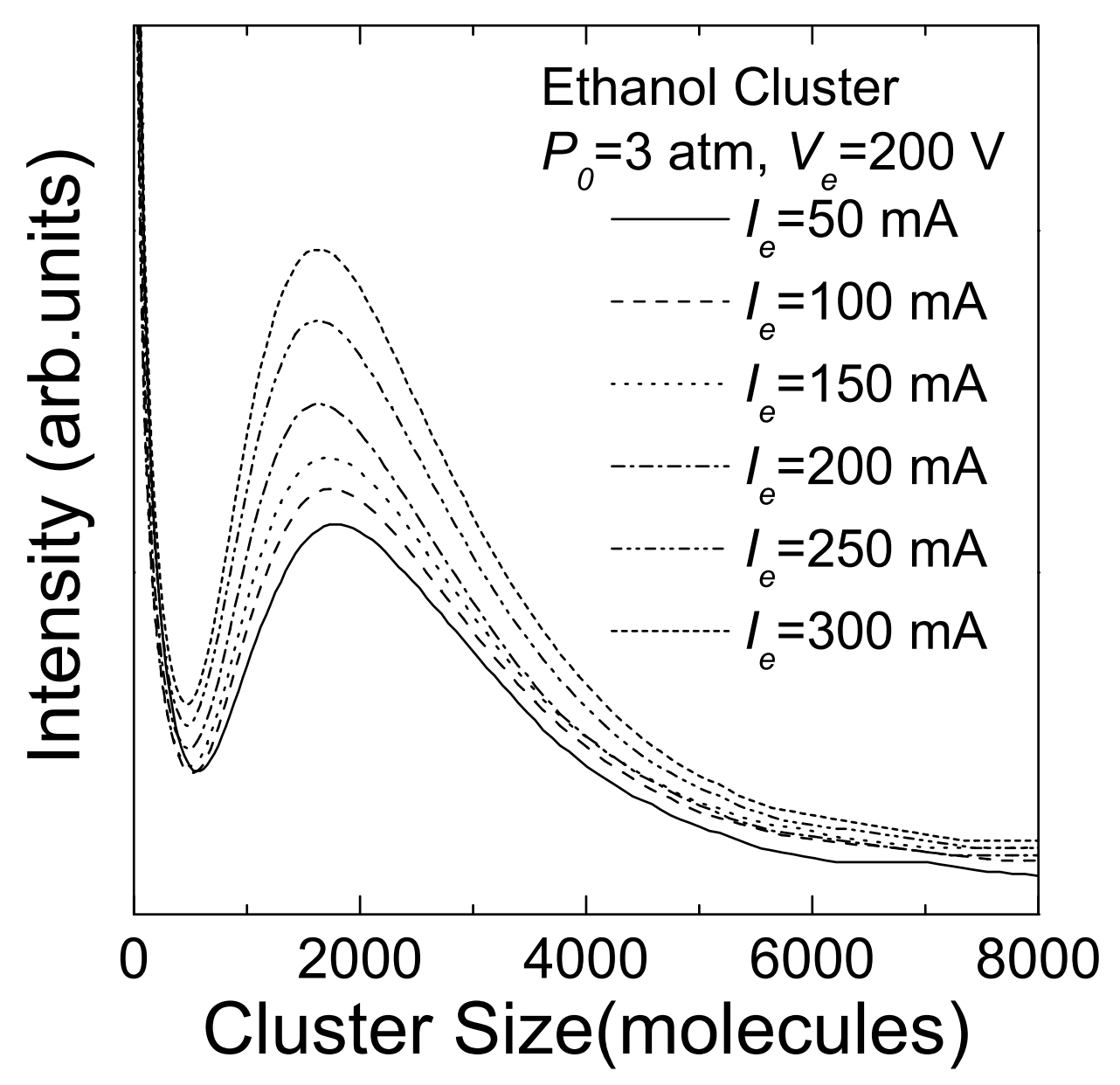

Fig. 4. 


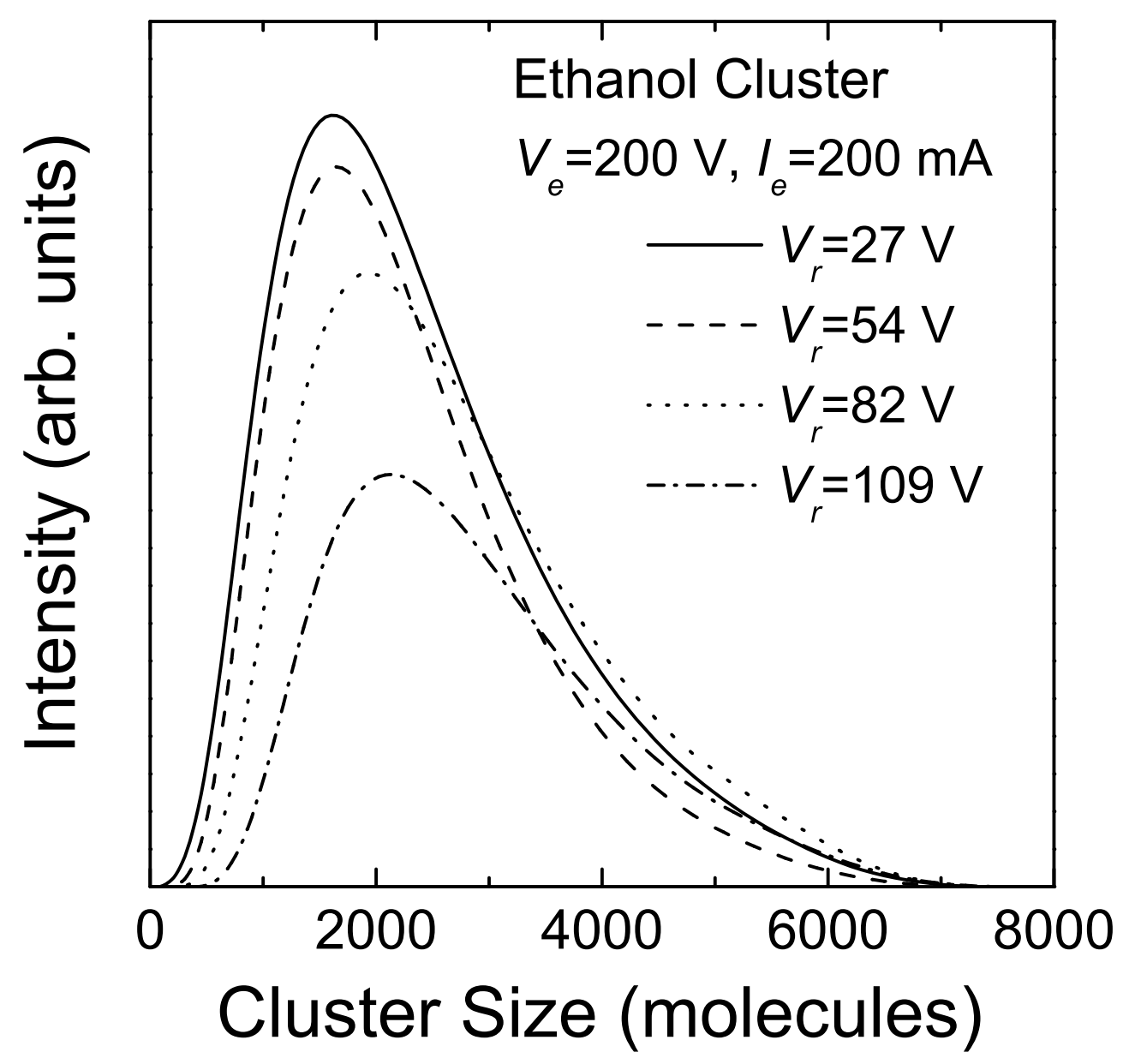

Fig. 5 . 


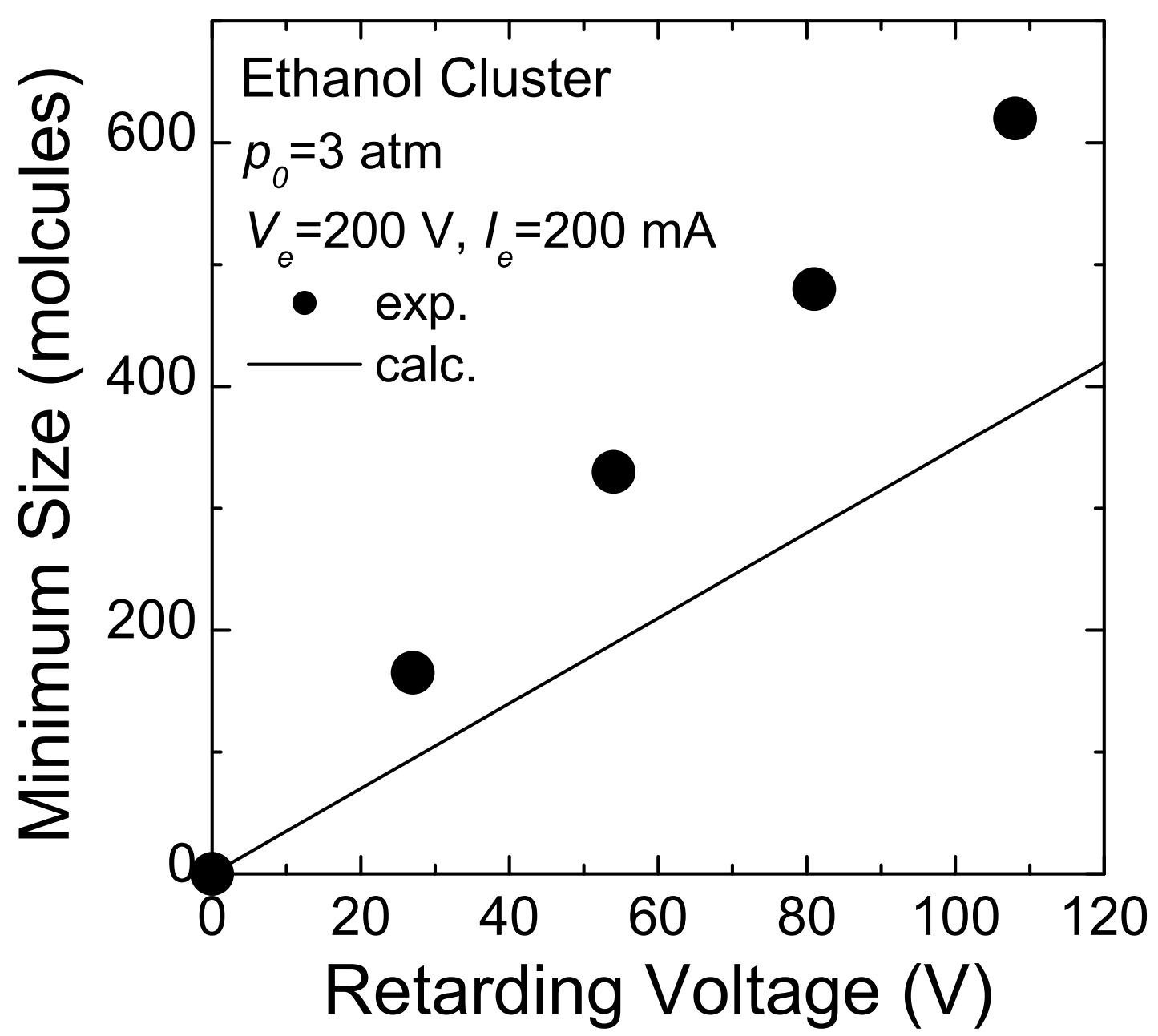

Fig. 6 . 